\title{
A influência de atividades de dupla tarefa na cinemática da marcha de indivíduos com hemiparesia espástica
}

\author{
The influence of dual task activities in the gait kinematics of individuals with spastic \\ hemiparesis
}

\author{
Tamara Luzia Primel Sául ${ }^{1}$, Joice Enriconi ${ }^{1}$, Leandro Viçosa Bonetti², Raquel Saccani ${ }^{3 *}$ \\ ${ }^{1}$ Graduação em Fisioterapia pela Universidade de Caxias do Sul (UCS); ${ }^{2}$ Graduação em Fisioterapia pela \\ Universidade Federal de Santa Maria (UFSM), Doutor em Neurociências pela Universidade Federal do Rio Grande \\ do Sul (UFRGS), Professor do Programa de Pós Graduação em Ciências da Saúde da Universidade de Caxias do Sul \\ (UCS); ${ }^{3}$ Graduação em Fisioterapia pela Pontifícia Universidade Católica do Rio Grande do Sul (PUCRS), Doutora em \\ Ciências do Movimento Humano pela Universidade Federal do Rio Grande do Sul (UFRGS), Professora do Curso de \\ Fisioterapia da Universidade de Caxias do Sul (UCS).
}

\begin{abstract}
Resumo
Objetivo: analisar a influência de atividades de dupla tarefa na cinemática da marcha de indivíduos com hemiparesia espástica. Metodologia: amostra de 12 indivíduos, com idade entre 30 e 71 anos. Primeiramente, as tarefas simples foram realizadas: marcha simples (tarefa motora), tarefa de fluência verbal (tarefa cognitiva) e tarefa visuoespacial (tarefa cognitiva). Após, as duplas tarefas foram realizadas, onde foi realizada a marcha ao mesmo tempo das tarefas cognitivas. Para a avaliação da marcha, foi utilizado um sistema de captura da trajetória tridimensional da marcha e as variáveis estudadas foram cadência, velocidade, comprimento da passada, largura do passo, tempo da passada, apoio simples e apoio duplo. Para a análise dos resultados utilizou-se estatística descritiva, o teste t pareado e o teste de Correlação de Pearson $(p<0,05)$. Resultados: na comparação com a marcha simples, durante as atividades de dupla tarefa os indivíduos apresentarem piores resultados nas variáveis cinemáticas da marcha. Durante a dupla tarefa de fluência verbal, as principais diferenças quando comparado com a marcha simples foram: velocidade, cadência, o tempo da passada, comprimento da passada e apoio simples. Já a dupla tarefa cognitiva visuoespacial demonstrou as principais diferenças na: velocidade, comprimento da passada e apoio simples. Houve correlação forte e significativa do Mini Exame do Estado Mental com a tarefa de fluência verbal simples e com a dupla tarefa; assim como para a tarefa visuoespacial simples e na dupla tarefa. Conclusão: conclui-se que ocorreu alteração em alguns parâmetros cinemáticos da marcha quando associado à dupla tarefa, com maior influência negativa da tarefa cognitiva de fluência verbal quando comparadas com a visuoespacial.
\end{abstract}

Palavras-chave: Hemiparesia. Marcha. Cognição.

\begin{abstract}
Objective: o analyze the influence of dual task activities on gait's kinematics in individuals with plastic hemiparesis. Methodology: included 12 individuals, aged between 30 and 71 years old. Firstly, simple tasks were performed: simple gait (motor task), verbal fluency task (cognitive task) and visuospatial task (cognitive task). Afterwards, the dual tasks were performed, where the gait was performed at the same time as the cognitive tasks. For gait assessment, a three-dimensional gait trajectory capture system was used and the variables studied were cadence, velocity, stride length, stride width, stride time, single support and double support. Descriptive statistics, paired $t$-test and Pearson's correlation test were used to analyze the results $(p<0.05)$. Results: in comparison with single gait, during dual task activities, individuals showed worse results in gait's kinematic variables. During the dual task of verbal fluency and gait, the main differences when compared to simple gait were: velocity, cadence, stride time, stride length and simple support. Already visual-spatial cognitive task and gait demonstrated the main differences in: velocity stride length, simple support. There was a strong and significant correlation between Mini Mental State Examination with the simple verbal fluency task and with the double task; as well as for the simple visuospatial task and with the double task. Conclusion: it was concluded that modifications on some gait's kinematic parameters of gait occurred when associated with the double task, with a greater negative influence of the cognitive task of verbal fluency when compared with the visuospatial task.
\end{abstract}

Keywords: Hemiparesis. Gait. Cognition.

\section{INTRODUÇÃO}

A hemiparesia espástica é um dos déficits resultantes de uma Lesão Encefálica Adquirida (LEA); sendo caracte-

Correspondente/Corresponding: *Raquel Saccani - End: Rua Francisco Getúlio Vargas, 1130, Bloco 70, Caxias do Sul, RS, Brasil, CEP 95070-560. - Tel: +55 (54) 3218-2774. - E-mail: rsaccani@ucs.br rizada como um acometimento que ocorre no encéfalo maduro e não relacionado a doenças hereditárias, congênitas e degenerativas. $O$ acidente vascular encefálico (AVE), traumatismo crânio encefálico (TCE) e tumores do sistema nervoso central (SNC) são os exemplos principais que compõem o grupo das LEA (SILVA; NEVES, 2009). 
A marcha hemiparética é uma das consequências mais comuns após LEA e geralmente caracterizada por um padrão assimétrico de andar associado à fraqueza motora contralateral ao hemisfério cerebral acometido (WESTPHAL et al., 2016). Geralmente ocorrem déficits no controle motor, sensorial e/ou perda proprioceptiva que interferem na locomoção, reduzindo a capacidade nas atividades de vida diária, uma vez que, surgem diferentes alterações na funcionalidade (BOWEN et al., 2001). O padrão da marcha hemiparética apresenta variações devido ao tipo de acometimento neurológico e também ao grau de espasticidade muscular presente no indivíduo. Ainda, a presença de alterações cognitivas, comuns nos pacientes com LEA, potencializa as dificuldades na execução de tarefas motoras (BALDAN; ELMAUER, 2015), pois déficits cognitivos estão associados a diminuição da mobilidade e risco aumentado de quedas (BONETTI et al., 2019).

Apesar dessas alterações e das dificuldades enquanto caminham, indivíduos hemiparéticos normalmente realizam duas atividades ao mesmo tempo (PLUMMER-D'AMATO; ALTMANN; REILLY, 2011). A realização de duas atividades, com objetivos distintos, de forma simultânea é chamada de atividade de dupla tarefa (DT), modelo muito utilizado nos últimos anos para avaliar a marcha e a cognição, ou seja, a correlação e os efeitos entre ambas (BALDAN; ELMAUER; 2015). A DTs são frequentes na vida cotidiana, como andar tomando um café ou falando no celular (LIU et al., 2017). Por isso, o estudo das atividades de DT são importantes, pois revelam as anormalidades associadas ao controle motor da marcha não evidentes em testes de tarefa motora simples, justificando o interesse por aplicações clínicas dos testes de dupla tarefa (SIMONI et al., 2013).

O desempenho durante atividades duplas pode ser um item importante tanto na abordagem avaliativa quanto para programas de reabilitação motora, já que estas atividades estão presentes na vida diária dos indivíduos; e o seu treinamento pode melhorar a função e mobilidade dos pacientes (KIM; HAN; LEE, 2014). Recentes estudos com DT demonstraram que indivíduos após AVE possuem dificuldade na realização de atividades de origem cognitiva, e consequentemente, apresentam capacidade motora reduzida durante atividades de tarefa dupla (LIU et al., 2017; MEESTER et al., 2019; YANG et al., 2007). Além disso, a natureza da tarefa cognitiva demonstra impactar de forma significativa no desempenho dos pacientes (DEBLOCK-BELLAMY; LAMONTAGNE; BLANCHETTE, 2020). Entretanto, há poucos estudos que avaliam a interferência de diferentes atividades de dupla tarefa em vários parâmetros cinemáticos da marcha. Ainda, a maioria dos estudos limitam-se a avaliação de pacientes após AVE e em ambiente clínico comum, o que torna os resultados mais suscetíveis a erro, pois não conseguem descrever com propriedade as múltiplas variáveis da marcha que sofrem alterações na DT. Por isso, o objetivo deste estudo foi analisar a influência de atividades de dupla tarefa na cinemática da marcha de indivíduos com hemiparesia espástica.

\section{METODOLOGIA}

A presente pesquisa se caracteriza como descritiva e observacional de caráter comparativo e correlacional, com abordagem transversal. Este estudo foi aprovado (número do parecer 3.114.517) pelo Comitê de Ética e Pesquisa da Universidade de Caxias do Sul (Caxias do Sul, Rio Grande do Sul, Brasil) e conduzido de acordo com a Resolução no 466 de 12 de dezembro de 2012, do Conselho Nacional de Saúde, que aprova as diretrizes e normas regulamentadoras de pesquisas envolvendo seres humanos. O local de realização deste estudo foi o Laboratório de Análise Biomecânica do Movimento Humano do Centro Clínico da Universidade de Caxias do Sul (CECLIN-UCS), localizado no Bloco 70, da Universidade de Caxias do Sul (Caxias do Sul, Rio Grande do Sul, Brasil).

Fizeram parte da amostra 12 indivíduos, maiores de 21 anos, com idade entre 30 e 71, residentes da Serra Gaúcha, cadastrados no CECLIN-UCS. O número amostral foi estabelecido por conveniência, determinado de forma intencional e não probabilística, de acordo com o número de pacientes e disponibilidade dos mesmos para participação na pesquisa. Foram incluídos os indivíduos: a) cadastrados na CECLIN-UCS; b) com hemiparesia espástica após uma LEA; c) que caminhassem de forma independente (critério de locomoção 6 ou 7, conforme o instrumento Medida de Independência Funcional - MIF); d) com capacidade para a realização das tarefas cognitiva e visuo-espacial; e) com tratamento fisioterápico finalizado nos últimos 5 anos ou que estivessem em fase final de reabilitação; f) que assinassem o Termo de Consentimento Livre e Esclarecido (TCLE). Foram excluídos os pacientes que: a) apresentassem hemiparesia flácida (hipotonia), tetraparesia, paraparesia, plegias e que apresentassem afasia de Wernicke; b) apresentassem deficiência visual e auditiva sem correção; c) apresentassem instabilidade cardiovascular, neurológica, musculoesquelética ou outra(as) condição(ções) clínica(s) instável(eis) que interferisse na marcha independente e/ou na realização da avaliação da marcha de forma segura; d) apresentassem déficits cognitivos que interferissem no entendimento sobre o TCLE, nos questionários e/ou no entendimento do protocolo de coleta de dados da marcha; e) relatassem alguma dor aguda incapacitante no momento da avaliação; f) tivessem idade inferior a 21 anos.

A escolha da população de indivíduos com hemiparesia espástica após LEA ocorreu por se tratarem de lesões neurológicas muito incidentes em serviços de reabilitação, havendo escassez de estudos com pacientes hemiparéticos por outras lesões além AVE. Ressalta-se que embora se reconheça que a heterogeneidade de alterações encefálicas possa estar associada a intensidade e modulações diferentes de alterações cognitivas e motoras, os critérios de exclusão estabelecidos procuraram tornar a amostra mais homogênea.

Primeiramente, foi realizada uma busca no banco de dados do Centro Clínico da UCS, com o intuito de 
identificar indivíduos que se encaixavam nos critérios de inclusão e exclusão do estudo. Após, foi realizado contato telefônico com os indivíduos selecionados, com o objetivo de confirmar se estes se enquadram nos critérios do estudo e verificar se havia interesse destes em participar da pesquisa. Para os que aceitaram participar da pesquisa e que se enquadraram nos critérios para participação, foi agendada uma avaliação no Laboratório de Análise Biomecânica do Movimetno Humano do Centro Clínico, localizado no Bloco 70 da Universidade de Caxias do Sul. No dia agendado para a avaliação, os participantes foram recebidos no Laboratório de Marcha, tiveram explicações sobre a pesquisa, sobre os procedimentos de coleta de dados e receberam o TCLE. Em caso de dúvidas, estas foram sanadas pelos pesquisadores. Os participantes que concordaram em participar do estudo assinaram o TCLE e, em seguida, passaram por um processo de triagem.

Inicialmente foi aplicado o questionário sobre dados pessoais e informações sobre a patologia. Na sequência, foi realizada a aplicação do Mini Exame do Estado Mental (MEEM), que é considerado uma avaliação padronizada, simplificada e rápida, com ampla aceitação na comunidade científica e clínica, já validado e adaptado para a população brasileira (BRUCKI et al., 2003). O MEEM avalia funções cognitivas como orientação espacial, temporal, memória imediata e de evocação, cálculo, linguagem-nomeação, repetição, compreensão, escrita e cópia de desenho. 0 escore máximo é de 30 pontos e escores menores que 23 podem ser interpretados como dano cognitivo (BRUCKI et al., 2003). No presente estudo, o MEEM foi utilizado como critério de caracterização, tendo como análise principal os domínios de "orientação espacial" (5 pontos) e "orientação temporal" (5 pontos), "registro de três palavras" ( 3 pontos), "recordação" de 3 palavras ( 3 pontos) e "linguagem" - itens "nomear dois objetos" e "comando de estágios" (2 e 3 pontos respectivamente). Os domínios "atenção e cálculo" (5 pontos), os itens "ler" e "escrever" do domínio "linguagem" (1 ponto cada) e o domínio "capacidade construtiva visual" (1 ponto) não foram avaliadas por serem consideradas irrelevante para caracterização da amostra no presente estudo. Ainda, alguns indivíduos apresentavam acometimento no hemicorpo dominante, perdendo a habilidade de motricidade fina para tarefas como escrever e desenhar e também por alguns indivíduos não serem alfabetizados, dificultando o domínio de ler, escrever e cálculo.

$\mathrm{Na}$ sequência, a MIF foi aplicada através de conversa com paciente. É um instrumento de avaliação quantitativa referente a incapacidade de pacientes com restrições funcionais em tarefas motoras e cognitivas de vida diária. Estão descritos dois domínios na MIF, o motor e o cognitivo. As atividades avaliadas estão divididas entre autocuidado, transferências, locomoção, controle esfincteriano, comunicação e cognição social, que inclui memória, interação social e resolução de problemas. Cada uma das atividades recebe uma pontuação que parte de 1 (dependência total) a 7 (independência completa), assim a pontuação total varia de 18 a 126 (RIBERTO et al., 2004).

Posteriormente, foi realizada a aplicação da escala de Ashworth modificada que segundo Alorani et al. (2015) é um método quantitativo utilizado na prática clínica para avaliação do tônus muscular. Caracteriza-se por uma graduação da resistência muscular encontrada durante o alongamento passivo de forma rápida, onde essa graduação varia de 0 (tônus normal) à 4 (articulação rígida).

Após a aplicação destes testes e questionários, ocorreu uma verificação minuciosa dos dados obtidos, comprovando se os mesmos seguiam os critérios de inclusão. Os indivíduos que seguissem os critérios, passaram pelas mensurações dos dados antropométricos, como peso e altura e a aferência dos sinais vitais.

Finalizada a primeira etapa, os participantes da pesquisa realizaram, de maneira randômica, as tarefas simples (TSs): a tarefa cognitiva de fluência verbal, a tarefa cognitiva visuoespacial e a tarefa motora da marcha simples. Para a realização das tarefas cognitivas, os participantes foram convidados a sentar em uma cadeira confortável, em uma sala silenciosa. Na tarefa cognitiva de fluência verbal, os participantes falavam em voz alta o máximo de palavras que começassem com as letras $P$ ou B, durante 1 minuto. Já durante a tarefa visuoespacial, o indivíduo se manteve sentado confortavelmente e foi instruído que após o comando verbal, deveria identificar imagens fixadas na parede. Esta atividade aconteceu através de uma sequência com três formas geométricas (quadrado, triângulo e círculo), de cores diferenciadas (amarelo, verde e azul), fixadas em diferentes lateralidades (direita e esquerda) e alturas (superiormente e inferiormente). O paciente deveria realizar o comando, como por exemplo: direcionar a cabeça para o quadrado amarelo à sua direita ou direcionar a cabeça para o círculo azul à sua esquerda, localizando se está fixado mais inferiormente ou superiormente. Após o comando verbal, o avaliador verificava se a identificação estava correta para obter pontuação na tarefa, dentro de um período de 60 segundos. Posterior a quantificação nas ações isoladas, tomou-se por nota e o indivíduo foi direcionado para a realização da marcha.

Para a realização da marcha simples, os procedimentos para coleta de dados foram baseados no protocolo de Laroche et al. (2011). Para adaptação dos participantes ao protocolo de avaliação, primeiramente foi solicitado aos sujeitos que caminhassem 8 metros em linha reta, na velocidade auto selecionada, no local destinado à coleta de marcha no laboratório. Após, foram afixados marcadores reflexivos nos seguintes pontos anatômicos, a direita e esquerda: espinhas ilíacas ântero-superiores, espinhas ilíacas póstero-superiores, porções médio-lateral dos fêmures, porções médio-lateral dos joelhos, porções médio-lateral das tíbias, maléolo lateral dos tornozelos, porções centro-posterior dos calcâneos e face dorsal dos segundos metatarsos, como observado na figura 1. Foram realizadas diversas tentativas até que 8 passos foram capturados integralmente. Para a captura da trajetória 
tridimensional dos marcadores posicionados no corpo dos sujeitos durante a marcha foi utilizado um sistema de cinemetria dotado de 7 câmeras integradas (VICON MX systems, Oxford Metrics Group, Reino Unido). Os dados cinemáticos foram coletados em uma taxa de amostragem de $100 \mathrm{~Hz}$.

Figura 1 - Afixação de marcadores reflexivos em pontos anatômicos.
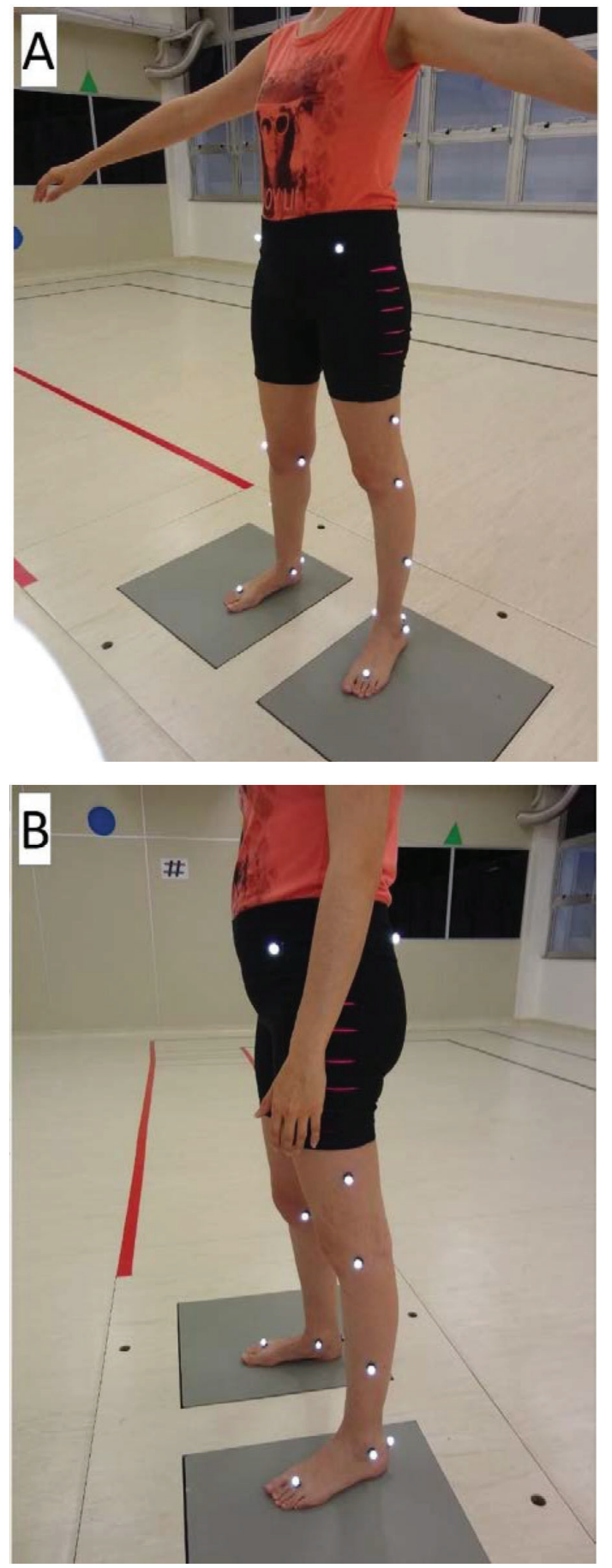

Legenda: Figura A: afixação de marcadores reflexivos vista frontal. Figura B: afixação de marcadores reflexivos vista sagital.

Fonte: Autoria própria
Após a realização de cada uma das tarefas simples, foram realizadas as atividades de DTs. As DTs consistiram na realização da tarefa motora da marcha realizada simultaneamente à cada uma das tarefas cognitivas; isto é, a marcha em velocidade auto selecionada foi realizada ao mesmo tempo que cada uma das 2 tarefas cognitivas. Para a análise dos resultados cognitivos durante as DTs, o tempo do primeiro minuto foi considerado para análise. Vale ressaltar que, assim como as TSs, durante a realização das DTs, a ordem de execução das atividades dessas DTs foi definida de forma randômica, através de um sorteio realizado pelos pesquisadores antes da chegada do indivíduo para a realização da pesquisa. Cabe ressaltar que durante a aplicação das tarefas, não foi avaliada a dupla tarefa motora, porque muitos sujeitos utilizavam dispositivo auxiliar no membro sadio ( $50 \%$ da amostra) e possuíam muitas limitações funcionais no membro afetado, impossibilitando a realização de outra tarefa motora com os membros superiores.

Toda a avaliação foi realizada no mesmo dia, com duração de aproximadamente 1 hora e 30 minutos, havendo intervalos de descanso sempre que houvesse necessidade. Destaca-se que as avaliações sempre foram realizadas pelas mesmas pesquisadoras e a análise da marcha pelo técnico do laboratório.

Os dados coletados foram analisados através do programa estatístico SPSS 21.0 (Statistical Package to Social Sciences for Windows). Para a descrição das variáveis referentes à caracterização da amostra, cinemática da marcha e dos desempenhos cognitivos, durante as TSs e durante às DTs, foi utilizada estatística descritiva com distribuição de frequência simples e relativa, bem como as medidas de tendência central (média) e de variabilidade (desvio padrão). Para a análise dos resultados da marcha e das tarefas cognitivas, comparações entre as TSs e as DTs empregou-se o teste t pareado. Para as correlações, foi usado o teste de Correlação de Pearson. Como critério de decisão, o nível de significância adotado foi $p \leq 0,05$. Para as correlações foram consideradas correlações fortes os valores acima de 0,60.

\section{RESULTADOS}

Foram avaliados 12 indivíduos, sendo que nove apresentavam hemiparesia espástica após AVE, dois indivíduos após ressecção tumoral e um deles após TCE, sendo $75 \%$ (nove participantes) da amostra composta pelo sexo feminino e $25 \%$ (três participantes) do sexo masculino. Desses 12 participantes, seis apresentaram alteração no hemicorpo esquerdo e seis no hemicorpo direito. Sobre a utilização de dispositivos auxiliares para deambulação, seis integrantes utilizavam bengalas de quatro pontos ou muletas canadenses ( $50 \%$ da amostra) e 6 possuíam marcha independente ( $50 \%$ da amostra), sem auxílio de dispositivos.

Em relação à avaliação de espasticidade, a maioria dos indivíduos apresentou padrão extensor de membros 
inferiores (Msls) e flexor de membros superiores (MsSs), com variabilidade entre eles (três pacientes apresentaram aumento de tônus apenas em gastrocnêmio e sóleo). Dos 11 participantes avaliados, apenas um demonstrou padrão flexor em joelho, apresentando espasticidade em isquiotibiais. Quanto ao padrão extensor de Msls (aumento de tônus em plantiflexores, extensores de joelho, flexores e adutores de quadril), todos, sem nenhuma exceção, apresentaram espasticidade em plantiflexores, variando de um a três, de acordo com a escala de Asworth modificada.

$\mathrm{Na}$ tabela 1, estão descritas as características dos pacientes e testes funcionais. Os indivíduos tinham idades entre 30 e 71 anos e o IMC alternou entre indivíduos classificados com baixo peso a obesos. Em relação à MIF, observou-se diferença nos itens avaliados, obtendo classificação de dependência e independência, demonstrando grande variabilidade funcional entre os pacientes. Conforme tabela, no MEEM os indivíduos também apresentaram variabilidade na capacidade cognitiva (dentro dos domínios avaliados), não sendo delimitado um ponto de corte (YANG et al., 2007). O tempo de lesão e de reabilitação fisioterapêutica variaram entre 3 meses e 180 meses.

Tabela 1 - Caracterização da amostra dos 11 indivíduos com hemiparesia espásticas após (EA). Caxias do Sul, RS, julho a novembro de 2019.

\begin{tabular}{lrrrr}
\hline \multicolumn{1}{c}{ Características } & Média & DP & Min & Max \\
\hline Idade (anos) & 48,25 & 12,86 & 30 & 71 \\
Altura (m) & 1,61 & 0,10 & 1,50 & 1,81 \\
Peso (kg) & 69,67 & 14,60 & 49,90 & 91,40 \\
IMC (kg/m²) & 27,10 & 7,02 & 17,50 & 37,90 \\
MIF (p) & 112,67 & 20,24 & 58 & 126 \\
MEEM (p) & 18,41 & 5,72 & 5 & 22 \\
Tempo de lesão (me) & 41,83 & 52,79 & 3 & 180 \\
Tempo de terapia (me) & 38,75 & 52,91 & 3 & 180 \\
\hline
\end{tabular}

Legenda: DP: desvio padrão; min: mínimo; max: máximo; m: metros; $k$ : quilograma p: pontos; Me: meses.

Fonte: Dados da pesquisa.

A tabela 2 apresenta os resultados da análise dos parâmetros lineares da marcha simples (MS), dupla tarefa fluência verbal (DTFV) e dupla tarefa visuoespacial (DTVE). Os indivíduos demonstraram diferenças estaticamente significativas na maioria das variáveis comparando a MS com as DTs, piorando o padrão de marcha ao realizar as tarefas combinadas. A DTFV gerou maior interferência na cinemática da marcha dos indivíduos avaliados. A tabela demonstra que nas DTs, os indivíduos foram mais lentos, tendo sua velocidade, cadência e comprimento da passada diminuídos, com aumento no tempo da passada e no apoio simples.
Tabela 2 - Dados lineares da marcha comparando a tarefa simples de marcha (MS) com a dupla tarefa fluência verbal (DTFV) e dupla tarefa visuoespacial (DTVE) de indivíduos com hemiparesia espástica após LEA. Caxias do Sul, RS, julho a novembro de 2019.

\begin{tabular}{|c|c|c|c|c|c|}
\hline \multirow{2}{*}{$\begin{array}{l}\text { Variáveis } \\
\text { lineares }\end{array}$} & MS & DTFV & $p$ & DTVE & $p$ \\
\hline & \multicolumn{2}{|c|}{$M d \pm D P$} & \multicolumn{3}{|c|}{$M d \pm D P$} \\
\hline $\begin{array}{l}\text { CAD } \\
\text { (passos/ } \\
\text { min) }\end{array}$ & $76,33 \pm 28,54$ & $66,15 \pm 24,76$ & $0,03 *$ & $69,46 \pm 24,12$ & 0,06 \\
\hline $\operatorname{VEL}(\mathrm{m} / \mathrm{s})$ & $0,53 \pm 0,36$ & $0,39 \pm 0,27$ & $0,004 *$ & $0,41 \pm 0,27$ & $0,01^{*}$ \\
\hline $\mathrm{CP}(\mathrm{m})$ & $0,80 \pm 0,28$ & $0,69 \pm 0,25$ & $0,001 *$ & $0,68 \pm 0,27$ & $0,001 *$ \\
\hline $\operatorname{LP}(m)$ & $0,23 \pm 0,06$ & $0,23 \pm 0,05$ & 0,37 & $0,24 \pm 0,55$ & 0,27 \\
\hline $\mathrm{TP}(\mathrm{s})$ & $1,94 \pm 1,14$ & $2,22 \pm 1,26$ & $0,002 *$ & $2,07 \pm 1,07$ & 0,06 \\
\hline AS (s) & $0,70 \pm 0,67$ & $1,04 \pm 0,93$ & $0,05^{*}$ & $0,94 \pm 0,74$ & $0,05^{*}$ \\
\hline$A D(s)$ & $0,61 \pm 0,32$ & $0,57 \pm 0,17$ & 0,44 & $0,56 \pm 0,21$ & 0,30 \\
\hline
\end{tabular}

Legenda: MS: tarefa simples de marcha; DTFV: dupla tarefa fluência verbal: DTVE, dupla tarefa visuoespacial; Md: média; DP: desvio padrão; p: valor de p; CAD: cadência; passos/min: passos por minuto;VEL: velocidade; $\mathrm{m} / \mathrm{s}$ : metros por segundo; $C P$ : comprimento da passada; m: metros; LP: largura do passo; TP: tempo da passada; s: segundos; AS: apoio simples; AD: apoio duplo; *: estatisticamente significativos.

Fonte: Dados da pesquisa

Na tabela 3 é possível observar os resultados dos testes cognitivos de fluência verbal e visuoespacial, apresentando diminuição quando comparados à TS, mas sem diferença significativa. Entretanto, houve correlação forte e significativa do MEEM com a tarefa simples de fluência verbal $(r=0,82 ; p=0,001)$, tarefa dupla de fluência verbal $(r=0,65 ; p=0,024)$, tarefa simples visuoespacial $(r=0,79$; $p=0,002)$ e a tarefa dupla visuoespacial $(r=1 ; p=0,000)$, indicando que quanto menor a capacidade cognitiva, pior foi o desempenho na realização da dupla tarefa.

Tabela 3 - Testes cognitivos comparando a tarefa simples cognitiva (TSC) com a dupla tarefa (DT) de indivíduos com hemiparesia espástica após uma LEA. Caxias do Sul, RS, julho a novembro de 2019.

\begin{tabular}{lccr}
\hline & TSC & DT \\
\multicolumn{1}{c}{ Teste Cognitivo } & \multicolumn{2}{c}{ Md \pm DP } & p \\
\hline Fluência verbal (np) & $5,83 \pm 4,82$ & $5 \pm 3,76$ & 0,27 \\
Visuoespacial (ni) & $10,58 \pm 7,35$ & $9,5 \pm 5,19$ & 0,41 \\
\hline
\end{tabular}

Legenda: TSC: teste cognitivo simples; DT: teste cognitivo com a marcha (dupla tarefa); p: valor de p; Md: média; DP: desvio; np:número de palavras; ni: número de imagens.

Fonte: Dados da pesquisa

\section{DISCUSSÃo}

Os resultados do presente estudo demonstraram que durante a realização das atividades de dupla tarefa os participantes demonstraram pior desempenho nos parâmetros da marcha quando comparado à tarefa simples da marcha.Várias pesquisas já demonstraram que a realização de atividades de DT geram alterações na marcha, tanto em sujeitos saudáveis jovens e idosos (SIMONI et al., 2013; BONETTI et al., 2019), assim como 
em indivíduos com alterações neurológicas como o AVE indicando comprometimento na locomoção ao realizar simultaneamente duas tarefas com objetivos diferentes (BALDAN; ELMAUER, 2015; DEBLOCK-BELLAMY; LAMONTAGNE; BLANCHETTE, 2020; LIU et al., 2017; MEESTER et al., 2019; YANG et al., 2007). Em pacientes neurológicos, o comprometimento sensório-motor restringe a ação muscular e piora o controle corporal; e se a espasticidade estiver agindo sobre os membros inferiores, os déficits da marcha serão potencializados diante da falta de seletividade motora (BALDAN; ELMAUER, 2015). Além disso, conforme observado na caracterização dos indivíduos deste estudo, além das alterações motoras, muitos possuem déficits cognitivos que dificultam ainda mais a realização de atividades duplas (YANG et al., 2007).

Considerando as variáveis espaço-temporais, neste estudo observou-se a diminuição da velocidade e da cadência da marcha com demanda cognitiva em comparação com a marcha simples, corroborando com pesquisas prévias já realizadas (BOWEN et al., 2001; DA SILVA et al., 2015; YANG et al., 2007). Entretanto, Da Silva et al. (2015) pressupõem que a cadência é menos sensível à alterações, pois ressaltam em seu estudo que os indivíduos reduziram a velocidade durante as DTs com demanda cognitiva, porém, mantiveram a cadência para a mesma atividade.

A diminuição da velocidade, apontada no presente estudo, indica que os indivíduos com LEA tendem a priorizar a precisão e a conclusão da tarefa, em vez de manter a velocidade de locomoção. Os dados confirmam o relatado em alguns estudos prévios, cujo grupo de indivíduos com hemiparesia tendia a andar mais devagar durante atividades duplas que envolviam a marcha somada à uma atividade cognitiva (DA SILVA et al., 2015; GOH et al., 2017; PATEL; BHATT, 2014). Embora a realização de atividades de DT que envolvam duas tarefas motoras possa alterar a velocidade, as DTs que envolvem uma atividade motora e uma atividade cognitiva interferem de maneira mais significativa na velocidade da marcha (DEBLOCK-BELLAMY; LAMONTAGNE; BLANCHETTE, 2020; GOH et al., 2017). Semelhante ao presente estudo, Bowen et al. (2001) e Muci et al. (2020) demonstraram que após um AVE, os indivíduos apresentaram alteração de equilíbrio e velocidade durante a realização de DTs, ocasionando comprometimento na funcionalidade motora.

Em relação ao comprimento da passada, observou-se diminuição significativa durante todas as DTs analisadas, sugerindo possível compensação para manter o equilíbrio. Yang et al. (2007) ao compararem o desempenho de três grupos em dupla tarefa motora, observaram diminuição da passada em ambos os grupos, indicando que a lesão neural gera decrécimos na marcha devido à maior dificuldade na realização de atividades simultâneas. Ainda, os indivíduos do presente estudo não demonstraram diferença significativa na largura do passo quando associado às atividades combinadas, concluindo que durante a execução da DT eles mantiveram a base constante. $A$ análise da marcha simples de pacientes hemiparéticos indica que essa variá- vel demonstra pouca alteração, se mantendo mesmo em pacientes com níveis variados de comprometimento no hemicorpo afetado (WESTPHAL et al., 2016).

Sobre as variáveis temporais, os pacientes desta pesquisa aumentaram o TP durante as DTs, como já relatado em estudo prévio (YANG et al., 2007). Ao realizar estudo com 45 pacientes, subdivididos em 3 grupos, com níveis funcionais diferentes, Yang et al. (2007), observaram alteração na cinemática da marcha e ressaltaram que o tempo da passada aumentava em ambos os grupos, com maior repercussão nos indivíduos mais comprometidos. Já sobre o tempo de $A S$ e $A D$, observou-se um aumento do AS e diminuição do $A D$, indicando possível diminuição do equilíbrio e da simetria corporal durante a DT. Diante das atividades de DT que tenham uma tarefa com demanda cognitiva, os pacientes parecem ter aumentado o tempo de apoio sobre o lado sadio, justificando o aumento do tempo de apoio simples. Isso é possível, pois a análise da marcha hemiparética indica que o tempo do passo tende a ser maior no hemicorpo acometido (WESTPHAL et al., 2016).

Em relação à comparação dos resultados entre ambas as tarefas realizadas, foi observado maior interferência da DTFV sobre as características da marcha. Os tipos de atividade cognitiva utilizados para avaliar a influência da dupla tarefa sobre a capacidade de realizar a marcha variam bastante (PATEL; BHATT, 2014). Plummer-D'Amato, Altmann e Reilly (2011) realizaram estudo semelhante com indivíduos jovens e idosos indicando que a fluência verbal produz maior interferência na marcha. Destacaram também que a fala espontânea é uma tarefa altamente exigente e impactante na marcha em idosos, pois além da velocidade que variou nos dois grupos, a duração da passada e a simetria da marcha também foram afetadas pela tarefa de fala nos idosos, mas não nos indivíduos mais jovens (PLUMMER-D'AMATO; ALTMANN; REILLY, 2011).

Cabe ainda ressaltar, sob os aspectos cognitivos, que o nível de cognição impactou no desempenho da DT, pois os indivíduos com maior capacidade cognitiva no MMEM demonstraram maior habilidade durante as DTs; o que já foi relatado em pesquisas prévias (YANG et al., 2007). Além disso, o desempenho cognitivo dos participantes não decaiu muito durante a realização das DTs, como observado nos parâmetros da marcha. Plummer et al. (2013), em estudo de revisão, destaca que durante atividades de marcha com uma tarefa cognitiva adicional, os indivíduos demonstram decréscimos maiores no desempenho motor; fato que também foi observado na presente pesquisa, pois a dupla tarefa repercutiu muito sobre os parâmetros da marcha, sem gerar tantas mudanças no desempenho cognitivo.

A execução de duas tarefas simultâneas é frequentemente utilizada pelos seres humanos no desempenho de diversas atividades cotidianas e por isso vem sendo utilizada na reabilitação (EQBAL et al., 2020; KIM; HAN; LEE, 2014). Eqbal et al. (2020) ressalta que a fisioterapia convencional e o treinamento de dupla tarefa melhoraram 
a capacidade de marcha de pacientes com AVE. Por isso, a aplicação de um programa de reabilitação envolvendo a marcha associada à outra tarefa pode promover uma melhora no desempenho de atividades combinadas. Essa abordagem pode auxiliar no tratamento de pacientes neurológicos, pois o estímulo simultâneo à cognição e ao controle motor, reflete na capacidade de deambulação e independência funcional dos indivíduos.

Apesar do número amostral pequeno, os resultados desta pesquisa fornecem parâmetros importantes em relação a cinemática da marcha e a influência da capacidade cognitiva durante as DTs. Além disso, podem servir como ferramenta de avaliação e criação de programas interventivos para pacientes com lesão neurológica. Sugere-se que mais estudos sejam desenvolvidos com indivíduos após LEA e que diferentes tarefas motoras e cognitivas sejam utilizadas durante as DTs. Ainda, a realização de ensaios clínicos randomizados poderia ampliar os resultados encontrados.

\section{CONCLUSÃO}

Este estudo apontou alterações nos parâmetros lineares da marcha, diminuição da velocidade, cadência e comprimento da passada, além de aumento no TP e AS de indivíduos com hemiparesia espática durante a realização de DTs. Ainda, observou-se que quanto menor a capacidade cognitiva, maiores são as dificuldades durante as atividades de DT. Os resultados sugerem que os pacientes após LEA alteram seu padrão de marcha para manter a função de deslocamento quando são expostos a outra tarefa simultânea.

\section{REFERÊNCIAS}

ALORAINI, S. M. et al. Assessment of spasticity after stroke using clinical measures: a systematic review. Disabil. Rehabil., Londres, v. 37, n. 25, p. 2313-2323, Feb. 2015.

BALDAN, A. M. S.; ELMAUER, J. C. Interferência da dupla tarefa no desempenho da marcha de indivíduos com hemiparesia pós AVE. J. Health Sci. Inst, São Paulo, p. 365-370, 2015.

BONETTI, L. V. et al. The effect of mental tracking task on spatiotemporal gait parameters in healthy younger and middle-and older aged participants during dual tasking. Exp. Brain Res., Berlin, v. 237, n. 12, p. 3123-3132, Sept. 2019.

BOWEN, A. et al. Dual-task effects of talking while walking on velocity and balance following a stroke. Age Ageing, Londres, v. 30, n. 4, p. 319323, Ago. 2001.

BRUCKI, S. et al. Sugestões para o uso do mini-exame do estado mental no Brasil. Arq. Neuropsiquiatr., São Paulo, v. 61, n. 3B, p. 777-781, set. 2003.

DA SILVA, J. M. et al. Efeitos da dupla tarefa com demanda motora e demanda cognitiva na marcha de sujeitos hemiparéticos pós AVC. Rev. Neurociênc., São Paulo, v. 23, n. 1, p. 48-54, mar. 2015.
DEBLOCK-BELLAMY, A.; LAMONTAGNE, A.; BLANCHETTE, A.K. CognitiveLocomotor Dual-Task Interference in Stroke Survivors and the Influence of the Tasks: A Systematic Review. Front. Neurol., Canadá, v. 11, p. 882, 2020.

$\mathrm{GOH}$, L. Y. et al. Effects of cognitive and motor tasks on the walking speed of individuals with chronic stroke. Medicine, Londres, v. 96, n. 9, Mar. 2017.

IQBAL, M. et al. Comparison of dual task specific training and conventional physical therapy in ambulation of hemiplegic stroke patients: A randomized controlled trial. JPMA, Paquistão, v. 2019, 2020.

KIM, G. Y.; HAN, M. R.; LEE, H. G. Effect of dual-task rehabilitative training on cognitive and motor function of stroke patients. J. Phys. Ther. Sci., Moroyama, v. 26, n. 1, p. 1-6, Feb. 2014.

LAROCHE, D. et al. Test-retest reliability of 3D kinematic gait variables in hip osteoarthritis patients. Osteoarthr. Cartil., Londres, v. 19, n. 2, p. 194-199, Feb. 2011.

LIU, Y. C. et al. Cognitive and motor dual task gait training improve dual task gait performance after stroke-A randomized controlled pilot trial. Sci. Rep., Nova York, v. 7, n. 1, p. 1-8, June 2017.

MEESTER, D. et al. A randomized controlled trial of a walking training with simultaneous cognitive demand (dual-task) in chronic stroke. Eur. J. Neurol., Oxford, v. 26, n. 3, p. 435-441, Oct. 2019.

$\mathrm{MUCl}$, B. et al. What are the factors affecting dual-task gait performance in people after stroke? Physiother. Theory Pract., London, p. 1-8, June 2020.

PATEL, P.; BHATT, T. Task matters: influence of different cognitive tasks on cognitive-motor interference during dual-task walking in chronic stroke survivors. Top. Stroke Rehabil., Frederick, v. 21, n. 4, p. 347-357, Dec. 2014.

PLUMMER, P. et al. Cognitive-motor interference during functional mobility after stroke: state of the science and implications for future research. Arc. Phys. Med. and Rehabil., Chicago, v. 94, n. 12, p. 25652574. e6, Dec. 2013.

PLUMMER-D'AMATO, P.; ALTMANN, L. J. P.; REILLY, K. Dual-task effects of spontaneous speech and executive function on gait in aging: exaggerated effects in slow walkers. Gait Posture, Oxford, v. 33, n. 2, p. 233-237, Feb. 2011.

RIBERTO, M. et al. Validação da versão brasileira da medida de independência funcional. Acta Fisiátrica, São Paulo, v. 11, n. 2, p. 72-76, ago. 2004.

SILVA, C. B.; NEVES, R. D. C. M. A. fisioterapia na lesão encefálica adquirida do adulto. In: MOURA, E. W. de et al. (Org.). Fisioterapia aspectos clínicos e práticos da reabilitação. 2. ed. São Paulo, SP: Artes Médicas, 2009.

SIMONI, D. et al. Different motor tasks impact differently on cognitive performance of older persons during dual task tests. Clin. Biomech., Bristol, v. 28, n. 6, p. 692-696, July 2013.

WALSHE, E. A. et al. Comparable walking gait performance during executive and non-executive cognitive dual-tasks in chronic stroke: a pilot study. Gait Posture, Oxford, v. 71, p. 181-185, June 2019.

WESTPHAL, P. J. et al. Análise cinemática da marcha em indivíduos com hemiparesia espástica após acidente vascular cerebral. Sci. Med., Porto Alegre, v. 26, n. 2, abr./jun. 2016.

YANG, Y. R. et al. Dual-task-related gait changes in individuals with stroke. Gait Posture, Oxford, v. 25, n. 2, p. 185-190, Feb. 2007.

Submetido em: 19/05/2021

Aceito em: 26/01/2021 